\title{
Mobilitätsdynamiken und Wissensarbeit - zum Wandel berufsbedingter zirkulärer Mobilität
}

\author{
Simone Strambach $\cdot$ Hendrik Kohl
}

Eingegangen: 13. August 2014 / Angenommen: 8. Juli 2015 / Online publiziert: 5. August 2015

(C) Springer-Verlag Berlin Heidelberg 2015

Zusammenfassung In der jüngeren Mobilitätsforschung wird festgestellt, dass mobile und komplexere Arbeitsund Lebensformen an Bedeutung gewinnen. Diese sind eng verbunden mit dem sozioökonomischen Strukturwandel zur Wissensökonomie und mit Veränderungen in der Organisation von Erwerbsarbeit. Vereinzelte empirische Erkenntnisse deuten darauf hin, dass „Wissensarbeitende" mobiler sind als andere Erwerbstätige. Die genauen Zusammenhänge zwischen wissensintensiven Tätigkeiten und berufsbedingter - insbesondere sogenannter zirkulärer - Mobilität sind aber bislang kaum systematisch beleuchtet worden. Durch die Zusammenführung der interdisziplinären Mobilitätsforschung und der räumlichen Innovationsund Wissensforschung können hier Synergiepotenziale genutzt werden, die es ermöglichen, die fluide raum-zeitliche Bindung von Wissensarbeit und ihre komplexen Implikationen für die berufsbedingte zirkuläre Mobilität differenzierter zu analysieren.

Schlüsselwörter Wissensökonomie - Wissensarbeit Berufsbedingte Mobilität · Raum-zeitliche Dynamik

H. Kohl, Dipl. Geogr. $(\bowtie)$

Geographisches Institut der Universität Göttingen,

Goldschmidtstr. 5,

37077 Göttingen, Deutschland

E-Mail: hendrik.kohl@geo.uni-goettingen.de

Prof. Dr. S. Strambach

Fachbereich Geographie Philipps-Universität Marburg,

Deutschhausstr. 10,

35032 Marburg, Deutschland

E-Mail: simone.strambach@staff.uni-marburg.de
Mobility Dynamics and Knowledge Work: The Change of Job-Related Circular Mobility

\begin{abstract}
An increasing importance of mobile and complex forms of working and living has been noticed in recent mobility research. These developments are closely linked to the socio-economic structural change toward a knowledge economy and to changes in the organization of employment. So far only few empirical results indicate that knowledge workers are more mobile than other employees. However, the relationship between knowledge-intensive activities and job-related - especially the so-called circular-mobility has not been systematically examined in-depth. Merging interdisciplinary mobility research with the spatial innovation and knowledge science seems to be a promising approach for utilizing synergy potentials that allow a more differentiated analysis of the fluid, spatiotemporal connection between knowledge work and its complex implications for the job-related circular mobility.
\end{abstract}

Keywords Knowledge economy · Knowledge work · Job-related mobility · Time-space dynamic

\section{Einleitung}

In der jüngeren interdisziplinären Mobilitätsforschung wird festgestellt, dass mobile Arbeits- und Lebensformen erheblich an Bedeutung gewonnen haben (Limmer und Schneider 2008; Kesselring und Vogl 2010a; Scheiner/ Blotevogel/Frank et al. 2013). Wenn auch die quantitative Relevanz noch nicht abschließend geklärt ist, so werden derzeit die gestiegene Mobilität und ihre gesellschaftlichen und räumlichen Implikationen unter anderem mit Konzepten der Multilokalität und Translokalität erforscht (Weichhart 
2009; Didero/Pfaffenbach 2014). Die räumlichen Bezüge der Mobilitätsformen scheinen unschärfer zu werden, da sie oft mehrere Orte umspannen und über Ländergrenzen hinweg erfolgen (Dittrich-Wesbuer/Kramer 2014). Veränderte gesellschaftliche, ökonomische und technologische Rahmenbedingungen im Zuge der Globalisierung werden dafür verantwortlich gemacht (Didero/Pfaffenbach 2014). Die zeitliche und räumliche Flexibilisierung von Erwerbsarbeit (Minssen 2012) als Folge der weiter fortschreitenden Dezentralisierung und vertikalen Desintegration von Produktionssystemen und Unternehmensorganisation in Verbindung mit zunehmender Individualisierung von Lebensstilen werden als wesentliche Ursachen für komplexere Arbeitsund Lebensformen und zunehmende räumliche Mobilität hervorgehoben (Limmer/Schneider 2008; Schneider/Meil 2008; Weichhart 2009; Kesselring/Vogl 2010a; Didero/Pfaffenbach 2014; Dittrich-Wesbuer/Kramer 2014).

In der Mobilitätsforschung weisen nur wenige Arbeiten bislang explizit auf die Zusammenhänge von wissensintensiver Arbeit und berufsbedingter zirkulärer Mobilität hin (siehe dazu genauer unten), obwohl die wissensbasierte Ökonomie als Analyseperspektive des globalen Strukturwandels inzwischen zunehmend in der ökonomischen, gesellschaftlichen und politischen Debatte etabliert ist. Forschungen, welche diese beiden Thematiken verbinden, beziehen sich vorrangig auf residenzielle, längerfristige Formen der Mobilität und beleuchten beispielsweise die internationale Migration von Hochqualifizierten oder Wissenschaftlern (Mahroum 2000; Beaverstock 2002; Trippl 2013) oder von Wissensarbeitenden in Agglomerationen und Ballungsräumen im Rahmen der Reurbanisierungsdebatte (Gornig/Mundelius 2012; Siedentop 2012). In Abgrenzung zu residenziellen Formen der berufsbedingten Mobilität, bei denen eine Verlagerung des Wohnortes stattfindet (Migration), werden unter b erufsbedingter $z$ irkulärer Mobilität räumlich-physische Bewegungen gefasst, bei denen in regelmäßigen Abständen zum Ausgangspunkt zurückgekehrt wird (Gather/Kagermeier/Lanzendorf 2008). In Bezug auf zirkuläre Bewegungen verdeutlichen vereinzelte quantitative empirische Studien zwar ein „Mehr“ an Mobilen unter den Hochqualifizierten und in wissensintensiven Branchen (Bonnet/Orain 2010; Giza-Poleszczuk/ Stec/Komendant et al. 2010), darüber hinaus werden jedoch keine differenzierteren Zusammenhänge $\mathrm{zu}$ räumlicher berufsbedingter zirkulärer Mobilität aufgezeigt.

Erkenntnisse aus der jüngeren wirtschaftsgeographischen Forschung zur raum-zeitlichen Dynamik von Innovationsprozessen unterstreichen demgegenüber die komplexe Räumlichkeit von Wissensdynamiken. Interaktionsprozesse, die auf Wissensaustausch und Wissenstransformation gerichtet sind, scheinen jenseits einer territorialen Fixierung durch fluide temporäre Organisationsformen und RaumZeit-Bindungen gekennzeichnet zu sein. Inwiefern dies aber
Einfluss auf die berufsbedingte zirkuläre Mobilität von Wissensarbeitenden hat, ist in diesem Forschungsstrang bislang noch nicht systematisch analysiert worden.

Den Kern von Wissensarbeit bilden wissensintensive, meist immaterielle Tätigkeiten, die im Vergleich zu materiellen bzw. zu Routinetätigkeiten durch Eigenschaften der Komplexität, Neuartigkeit und Kollaboration gekennzeichnet sind (Hube 2005; Kelter/Rief/Bauer et al. 2009). Die Folge dieser Eigenschaften sind fluide Raum-ZeitBindungen, die zur Dynamisierung und Flexibilisierung von berufsbedingter zirkulärer Mobilität führen. Vor dem Hintergrund der zunehmenden wissensbasierten Ökonomie scheint eine differenziertere Betrachtung der raum-zeitlichen Zusammenhänge von Wissensarbeit und dem Wandel berufsbedingter zirkulärer Mobilitätsformen längst überfällig. Eine solche Zusammenführung kann dazu beitragen, neue Erkenntnisse in Bezug auf berufsbedingte zirkuläre Mobilität von Wissensarbeitenden zu erzeugen und die Prozesse und Mechanismen in ihrer Vielschichtigkeit und Komplexität differenzierter und systematischer zu erfassen.

Der theoretisch-konzeptionelle Artikel führt die weitgehend getrennten Stränge der Mobilitätsforschung und der räumlichen Innovations- und Wissensforschung zusammen, um mögliche theoretische und empirische Synergiepotenziale zu erschließen. Im Mittelpunkt steht die übergeordnete Forschungsfrage, wie sich Wissensarbeit, die auf vielfältigen wissensintensiven Tätigkeiten basiert, auf berufsbedingte zirkuläre Mobilität auswirkt. Um sich dieser Frage zu nähern, wird zunächst herausgearbeitet, welche Erkenntnisse bislang in der Mobilitätsforschung zum Wandel berufsbedingter zirkulärer Mobilität diskutiert werden und in welchem Zusammenhang diese zur Wissensarbeit stehen (Kap. 2). Anschließend wird den Fragen nachgegangen, durch welche Strukturen und Prozesse Wissensarbeit gekennzeichnet ist (Kap. 3) und welche Mechanismen die raum-zeitliche Bindung von Wissensarbeit prägen (Kap. 4), um daraus Implikationen für den Wandel berufsbedingter zirkulärer Mobilität von Wissensarbeitenden abzuleiten (Kap. 5).

\section{Der Wandel berufsbedingter zirkulärer Mobilität in der interdisziplinären Mobilitätsforschung}

Der vielschichtige und facettenreiche Begriff der Mobilität gilt als eines der wesentlichen Charakteristika moderner Gesellschaften (Schöller-Schwedes/Rammler 2008; Voß 2010). Oftmals gilt nur derjenige, der mobil ist, in unserer heutigen Gesellschaft als fortschrittlich, leistungs- und zukunftsfähig (Voß 2010: 95). Vor allem der Anspruch, in beruflicher Hinsicht räumlich mobil zu sein, stellt viele Erwerbstätige vor eine große Herausforderung. Kesselring/ Vogl (2010a: 51) sprechen in diesem Zusammenhang von 
einem Mobilitäts-Imperativ in der Arbeitswelt, in der von immer mehr Beschäftigten das Mobilsein gefordert werde. Nicht nur die Arbeitswelt, auch die sozialen Beziehungen sind im Zuge von Internationalisierungs- und Globalisierungsprozessen räumlich disperser geworden. Physische Mobilitäten werden vermehrt mit weiteren Mobilitätsformen wie virtueller und telekommunikativer Mobilität ergänzt, welche in komplexer Weise ineinandergreifen (Larsen/Axhausen/Urry 2006).

Im Hinblick auf berufsbezogene Mobilität wird in der interdisziplinären Mobilitätsforschung in den letzten Jahren vermehrt der Wandel berufsbedingter zirkulärer Mobilitätsformen und die damit verbundenen zunehmenden sozialen, psychischen und körperlichen Belastungen diskutiert (Limmer 2005; Schneider/Rüger/Münster 2009; Kesselring/Vogl 2010a). Ein umfassendes und gemeinsames Verständnis des Begriffs ist allerdings bislang noch nicht vorhanden. In der geographischen Verkehrsforschung werden diese Phänomene vorrangig unter dem Stichwort des zweckgebundenen Verkehrs beleuchtet, zum einen als Berufspendlerverkehr und zum anderen als Geschäfts- und Dienstreiseverkehr (Schliephake/Schenk 2005). Frühere Arbeiten unterstreichen, dass der Berufspendlerverkehr festen Rhythmen folgt, die durch Regelmäßigkeit und wiederkehrende Muster wie tageszeitabhängige Stoßzeiten geprägt sind. Dem Geschäftsverkehr wird generell nur wenig Aufmerksamkeit gewidmet. Er gilt als sehr differenziert und schwierig erfassbar (Heineberg 2007: 211 ff.) und wird vorrangig aus einem touristischen Blinkwinkel unter dem Stichwort des Geschäftstourismus bzw. „Business Travel“" beleuchtet (Davidson/Cope 2003). Demzufolge entziehen sich geschäftliche und dienstliche Wege ohne Übernachtung der wissenschaftlichen Betrachtung bislang weitestgehend.

Quantitativ-empirische Arbeiten aus der räumlichen Mobilitäts- und Verkehrsgeneseforschung untersuchen die Veränderungen des allgemeinen Mobilitäts- bzw. Verkehrshandelns. Sie weisen auf die Ausdifferenzierung der berufsbedingten Mobilität und das Aufkommen komplexer zirkulärer Mobilitätsformen im Kontext von Arbeit, Freizeit und Familie sowie Lebensstil hin. Viele Einzelaspekte dieses Wandels sind dabei vergleichsweise gut untersucht und viele Phänomene durch ökonomische und gesellschaftliche Veränderungsprozesse erklärbar. Aspekte wie raum-zeitliche Muster und Strukturen alltäglicher, zirkulärer Mobilität in Kombination mit der Verkehrsmittelwahl stehen im Fokus dieser Forschungen (Lanzendorf/Scheiner 2004; Scheiner 2009). Berufsbedingte zirkuläre Bewegungen werden in der Regel in allgemeine Studien zum Mobilitäts- und Freizeitverhalten integriert (PTV AG/Fell/Schönfelder et al. 2000; Ohnmacht 2006) oder häufig ganz auf das Berufspendeln zwischen Wohnung und Arbeitsstätte reduziert (Einig/Pütz 2007; Haas/Hamann 2008). Erklärungsansätze des Wandels des Mobilitätsverhaltens setzen meist auf einer Makroebene der Veränderung von ökonomischen, gesellschaftlichen und politischen Rahmenbedingungen an (Gather/Kagermeier/ Lanzendorf 2008). So wurde durch den sektoralen Strukturwandel und den damit einhergehenden Bedeutungsgewinn von Dienstleistungen und weitreichenden Verbesserungen der Informations- und Kommunikationstechnologien (IuK) gemeinhin eine Entkopplung des Verkehrsaufkommens von der Wirtschaftsleistung erwartet. Diese Überlegungen haben sich jedoch als nicht zutreffend erwiesen (Lanzendorf/Scheiner 2004: 20 ff.). Dennoch ist im Bereich der Pendlerstrukturen eine Flexibilisierung der täglichen, wöchentlichen und saisonalen Rhythmen der Verkehrsteilnahme nachgewiesen. Tägliche Verkehrsspitzen des Berufsverkehrs schwächen sich dadurch in der Konsequenz mehr und mehr ab (Scheiner 2009). Erklärt wird dies durch veränderte Raum- und Siedlungsstrukturen und räumliche Entgrenzungsprozesse am Arbeitsplatz durch neue Möglichkeiten der Heim- und Telearbeit (Lanzendorf/Scheiner 2004: 16 ff.). Ebenfalls werden veränderte Lebensstile und die Verkettung von Freizeit und Arbeitsmobilität als Ursachen angeführt. Aufgrund der Makroperspektive wird das Mobilitätshandeln allerdings nicht genauer vor dem Hintergrund von Arbeits- und Tätigkeitskontexten analysiert. So bleibt beispielsweise offen, ob in wissensintensiven Arbeitskontexten die Flexibilisierung von Pendlermustern besonders verbreitet ist.

Arbeiten aus der soziologischen Mobilitätsforschung rücken stärker das Individuum und die Lebens- und Arbeitsumstände in den Fokus und untersuchen umfassend die Spezifika von mobilen und multilokalen Arbeits- und Lebensformen (Limmer 2005; Schneider/Meil 2008; Rüger/ Feldhaus/Becker et al. 2011). Starre, einfach strukturierte Muster werden vermehrt von komplexen Mobilitätsarrangements wie gesteigerter Umzugsmobilität, Fern- oder Wochenendpendeln (,shutteln') sowie dem häufigen Übernachten fernab der eigenen Wohnung (Varimobilität) abgelöst (Schneider/Meil 2008; Rüger/Feldhaus/Becker et al. 2011). In einer groß angelegten europaweiten Studie wurden mobile Lebensformen umfassend empirisch untersucht. Es wird betont, dass die untersuchten Mobilitätsformen unter allen Bevölkerungsschichten und Berufsgruppen verbreitet sind. Die Studienergebnisse zeigen jedoch auch, dass berufsbedingte zirkuläre Mobilitätsformen wie Fernpendeln, Wochenendpendeln (,Shutteln') und Varimobilität in wissensintensiven Branchen und Beschäftigungskontexten vermehrt vorkommen. So heben Giza-Poleszczuk/ Stec/Komendant et al. (2010) im Zuge der „JobMob and FamLives"-Studie die Einflüsse des sozialen Status, des Bildungsstandes und der beruflichen Position auf berufsbedingte zirkuläre Mobilitätsformen hervor. Die Ergebnisse weisen darauf hin, dass die Mobilen unter den Beschäftigten eher in den höheren sozialen Schichten vorzufinden sind und deren Anteile im wissensintensiven Sektor höher sind. 
Bonnet/Orain (2010: 292 ff.) stellen im Rahmen desselben Forschungsprojektes heraus, dass auffällig mehr Mobile als Fachkräfte in qualifizierten Positionen (,skilled positions“) arbeiten, die sich oftmals durch ein hohes Arbeitspensum und eine eher ungeregelte und flexiblere Arbeitsorganisation auszeichnen. Dies sind Hinweise auf einen Zusammenhang zwischen wissensintensiven Tätigkeiten und Mobilität. Allerdings setzen die verwendeten empirischen sozialen Variablen in diesem Forschungsprojekt in erster Linie auf dem Niveau von Qualifikationen an und können damit Wissensarbeit und die davon umfassten Tätigkeiten nur unzureichend abbilden. Weitgehend offen bleibt, welche Einflüsse Wissensarbeit auf die verschiedenen Erscheinungsformen berufsbedingter zirkulärer Mobilität hat und welche Mechanismen diesen Prozessen zu Grunde liegen.

Erkenntnisse aus der Multilokalitäts- und Translokalitätsforschung tragen ergänzend dazu bei, dass arbeitsbedingte, aber auch freizeit- und lebensstilbedingte multilokale Lebensformen an Bedeutung zunehmen (Weichhart 2009; Didero/Pfaffenbach 2014). „Steigende Mobilitätsanforderungen und die Flexibilisierung der Arbeitswelt mit einem Anstieg von kurzfristigen und instabilen Beschäftigungsverhältnissen bewirken, dass Arbeitnehmer und Selbstständige Arbeitsorte weit außerhalb ihrer Wohnorte aufsuchen und diese unter Umständen häufig wechseln müssen“ (Didero/ Pfaffenbach 2014: 5). Die empirische Erfassung von Multilokalität geht mit weitreichenden methodischen Problemen einher, die unter anderem dazu beitragen, dass es bisher kaum verlässliche Untersuchungen der Verbreitung dieser Phänomene gibt. Ob Wissensarbeitende von Multilokalität besonders betroffen sind und in welchem Zusammenhang dies mit ihren Tätigkeiten steht, ist noch weitgehend ungeklärt. Multilokale Wohnarrangements werden heute vor dem Hintergrund des Erhalts von (sozialräumlichen) Ankerpunkten interpretiert (Dittrich-Wesbuer/Kramer 2014). Inwieweit auch Erfordernisse wissensintensiver Tätigkeiten die Entstehung solcher Lebensstile beeinflussen und inwiefern dies Auswirkungen auf andere berufsbedingte zirkuläre Mobilitätsformen wie etwa den täglichen Arbeitsweg hat, wird kaum thematisiert.

Jüngere mobilitätssoziologische Forschungen nehmen stärker Dienst- und Geschäftsreisen in den Fokus und analysieren den Wandel von berufsbedingten Reisen und deren Konsequenzen für Betriebe und Beschäftigte (Kesselring/ Vogl 2010a; Kesselring/Vogl 2010b). Es wird herausgestellt, dass Dienst- und Geschäftsreisen in den letzten Jahren durch eine zunehmende räumliche Entgrenzung und zeitliche Verdichtung gekennzeichnet sind. Kesselring/ Vogl (2010b) gehen davon aus, dass permanentes Reisen zu einer Grundanforderung an das Arbeitsvermögen von immer mehr Beschäftigten werden wird. Sie belegen, dass nicht nur die Anzahl der Dienstreisen steigt, sondern auch eine Verdichtung der Reisetätigkeiten aufgrund einer Ver- kürzung der Reise- und Aufenthaltsdauer zu beobachten ist. Neben dem Trend zur stärkeren internationalen Vernetzung von Wirtschaftsunternehmen vor dem Hintergrund sich wandelnder Märkte und politischer und gesellschaftlicher Rahmenbedingungen wird hier auch die Einbettung der Reisetätigkeiten in institutionelle, betriebliche Kontexte hervorgehoben, die zu Rationalisierungstendenzen führt. Davon sind nicht nur Wissensarbeitende im engeren Sinne betroffen, sondern auch immer mehr Berufe, die ursprünglich nur wenig mobil waren, wie Facharbeiterinnen und Facharbeiter, kaufmännische Angestellte oder Laborangestellte. Allerdings stehen hier strukturelle Rahmenbedingungen auf sozialökonomischer und betrieblicher Ebene im Zentrum der Ausführungen und konkrete Bezüge zur Arbeit und den Tätigkeiten bleiben auch hier unberücksichtigt. Inwieweit Wissensarbeit dienstliche und geschäftliche Reisetätigkeiten beeinflusst und ob im Kontext wissensintensiver Arbeit sich neben einer Häufung und Verdichtung auch weitere raum-zeitliche Dimensionen der Mobilität verändern, wird hier nicht systematisch analysiert.

Darüber hinaus belegen Erkenntnisse aus Forschungen zu mobiler Arbeit, dass dieses Phänomen steigende Beachtung erfährt, wobei sich hier die Begrifflichkeiten teilweise mit den oben beschriebenen Aspekten überschneiden. Cohen (2010) untersucht aus einer arbeitssoziologischen Perspektive dieses Phänomen und unterscheidet drei Typen mobiler Arbeit: (1) „working while mobile“ als das Arbeiten, während man unterwegs ist, (2) „mobile for work" als die Mobilität, die zwar nicht den Kern der Arbeit darstellt, aber für die Arbeit notwendig ist und (3) ,mobile as work“, bei der die Mobilität die wesentliche Tätigkeit der Arbeit darstellt, wie beispielsweise bei Piloten oder Reisebegleitern. Auch bei Cohen steht die Wissensarbeit nicht im Zentrum der Analysen. Allerdings beschreibt Cohen das Arbeiten von unterwegs (working while mobile) als typisches Merkmal von Angestellten (,white-collar workers"). Dies weist auf die raum-zeitlichen Besonderheiten immaterieller Tätigkeiten hin. Die für die Arbeit notwendige Mobilität, die „,mobility for work", ist nach Cohen bislang nur wenig erforscht, was sich teilweise mit der Darstellung der bereits erläuterten Literaturstränge aus der Mobilitätsforschung deckt.

Als Ergebnis lässt sich festhalten, dass in den dargestellten Literatursträngen verschiedene Aspekte des Wandels berufsbedingter (nicht nur) zirkulärer Mobilität in hochentwickelten Gesellschaften auf unterschiedlichen Ebenen beleuchtet werden. Während einige Literaturstränge eher deskriptiv bleiben oder nur abstrakt den Wandel auf allgemeine wirtschaftliche und soziale Veränderungsprozesse zurückführen, wie etwa den technologischen Wandel und die fortschreitende Tertiärisierung der Ökonomie, treten in jüngerer Zeit vermehrt auch Arbeiten in den Vordergrund, welche als Erklärungsansätze des Wandels komplexere sozioökonomische Veränderungsprozesse in den Mittel- 
punkt stellen. Diese versuchen durch interdisziplinäre Forschung Erkenntnisse aus der Wirtschaftsgeographie und Arbeitssoziologie zum ökonomischen und sozialen Wandel zu integrieren (vgl. z. B. Schneider/Meil 2008; Kesselring/ Vogl 2010a; Kesselring/Vogl 2010b). Auf der Makroebene wird hier vereinzelt auch auf den wissensbasierten sozioökonomischen Strukturwandel Bezug genommen, in Folge dessen sich ein regional spezialisierter und dynamischer Arbeitsmarkt herausbildet, der beispielsweise für räumlich disperse und häufig wechselnde Arbeitsstätten verantwortlich ist. Hierdurch gewinnen zirkuläre Mobilitätsformen an Intensität (Schneider/Meil 2008; Schneider/Rüger/Münster 2009) und multilokale Lebens- und Wohnformen werden forciert (Didero/Pfaffenbach 2014).

Herausgestellt wird zudem, dass berufliche Reisetätigkeiten und mobile Arbeits- und Lebensformen keine Phänomene mehr sind, die einer akademischen und gesellschaftlichen Elite vorbehalten sind. Vielfältige Mobilitätserfahrungen werden heute von Beschäftigten in unterschiedlichen Tätigkeitsbereichen und auf unterschiedlichen Qualifikationsebenen gesammelt (Kesselring/Vogl 2010a: 46), wenngleich Hochqualifizierte und Beschäftigte in wissensbasierten Branchen durch quantitativ höhere Anteile in einzelnen Mobilitätsformen hervorstechen (Schneider/Ruppenthal/Lück et al. 2008; Bonnet/Orain 2010; Giza-Poleszczuk/Stec/Komendant et al. 2010). Im Detail sind die Auswirkungen und Einflüsse wissensintensiver Tätigkeiten auf berufsbedingte zirkuläre Mobilität jedoch noch wenig untersucht. Erste konzeptionelle und empirische Erkenntnisse in Bezug auf diese Zusammenhänge werden von Kohl (2014) aufgezeigt.

Das Fehlen von systematischen Untersuchungen an dieser thematischen Schnittstelle von Mobilität und Wissensarbeit ist unter anderem darauf zurückzuführen, dass das Phänomen der Wissensarbeit noch nicht eindeutig definiert ist und dass die interdisziplinäre Mobilitätsforschung und die räumliche Innovations- und Wissensforschung weitgehend getrennte Forschungsfelder sind. Aus Erkenntnissen der räumlichen Innovations- und Wissensforschung lassen sich Hinweise über Strukturen und Prozesse der Wissensarbeit und ihrer Raum-Zeitlichkeit ableiten und somit Potenziale erschließen, um die Implikationen für die berufsbedingte zirkuläre Mobilität differenzierter zu analysieren.

\section{Wissensproduktion in der Wissensökonomie - Strukturen und Prozesse der Wissensarbeit}

Die Generierung und Anwendung von Wissen aus der Sicht der Arbeitsteilung zu betrachten und damit die raum-zeitliche Dynamik einschließlich der verbundenen physischen Mobilität zu beleuchten, ist in der Debatte zur Wissensökonomie noch wenig ausgeprägt. „Wissensarbeit“" ist als ein begriffliches Konzept bis jetzt nicht einheitlich definiert.
Uneinigkeit besteht schon darin, was unter Wissensarbeit zu verstehen ist. Einige Autoren stellen die Aussagekraft des Begriffs „Wissensarbeit“ generell in Frage. Die Kritik an diesem Begriff betont einerseits, dass die Verrichtung von Arbeit immer mit Wissen und Kompetenzen verbunden war und ist. Andererseits wird bezweifelt, ob angesichts der enormen Vielfalt von Arbeitsinhalten und Kompetenzanforderungen von wissensintensiven Tätigkeiten diesen eine Gesamtheit unter dem Begriff der „Wissensarbeit“" zugeschrieben werden kann (Moldaschl/Stehr 2010; Minssen 2012; Moldaschl 2012).

Offensichtlich ist mittlerweile, dass die systematische Erzeugung, Kommerzialisierung und Kommodifizierung von Wissen sowie die reflexive Anwendung von Wissen auf die Wissensproduktion selbst zur Steigerung der immateriellen ökonomischen Wertschöpfung geführt hat und weiter führt. Substanziell empirisch fundiert ist inzwischen ebenfalls, dass mehr und mehr Anteile der Erwerbstätigen mit der Transformation von Wissen und der Produktion von neuem Wissen beschäftigt sind (UNESCO 2005; Dolfsma/ Soete 2006). Der Bedeutungszuwachs des Produktionsfaktors Wissen verläuft dabei quer über alle Sektoren und Branchen, wenngleich hier einige Wirtschaftsbereiche durch besonders hohe Dynamiken auffallen (Kujath 2010: 20 f.; Strambach 2011). Die vorhandenen statistischen Indikatoren, die sich primär auf Berufe, Wirtschaftszweige und Bildungsabschlüsse beziehen, lassen nur begrenzte Rückschlüsse auf den Wandel von Qualifikationsanforderungen und Kompetenzen zu, denen Erwerbstätige in wissensintensiven Tätigkeiten unterliegen. Die Suche nach neuen Zugängen und die Entwicklung neuer Ansätze stellen daher die Tätigkeiten selbst und die Anforderungen an Wissensarbeit in den Mittelpunkt. Über diese Merkmale wird Wissensarbeit von Nicht-Wissensarbeit abgegrenzt. Die explizite Unterscheidung zwischen Information und Wissen wird in Typologien berücksichtigt, mit denen in Wissensarbeit im engeren und weiteren Sinn unterschieden werden kann. Wissensarbeit im engeren oder originären Sinne hat dabei die Generierung von neuem Wissen zum Ziel, während Wissensarbeit im weiteren Sinn durch wissensaneignende oder wissensnutzende Tätigkeiten bestimmt wird, die vorhandene Informationen verknüpfen (Hube 2005: $63 \mathrm{f}$; Tiemann 2009: 7; Ibert/Kujath 2011: 13 f.).

Festhalten lässt sich derzeit, dass vorhandene Ansätze der Klassifizierung und Typisierung sich in ihrer Perspektive, der Schwerpunktsetzung und dem Abstraktions- und Aggregationsniveau unterscheiden, auf dem sie wissensintensive Tätigkeiten und Praktiken systematisieren (Hube 2005; Hall 2007; Tiemann 2009). Verantwortlich für die geringe Vergleichbarkeit der Systematisierungsansätze sind vor allem die besonderen Eigenschaften, die den zentralen Arbeitsinhalt kennzeichnen - die Erzeugung, Transformation und Anwendung von Wissen mit der Zielsetzung der 
ökonomischen Wertschöpfung. Sie sind der Grund dafür, dass die Arbeitsteilung bei der Wissensproduktion im Vergleich zu derjenigen der materiellen Produktion anderen ökonomischen Handlungslogiken und institutionellen Voraussetzungen unterliegt. Die Kontextabhängigkeit und Personengebundenheit, die implizite Dimension (Polanyi 1985), der Prozesscharakter, die Reflexivität und die Kumulativität von Wissen sind - wie im Folgenden gezeigt wird - dafür ausschlaggebend (Foray 2004; Antonelli 2005).

Im Unterschied zu Informationen ist Wissen nicht einfach übertragbar, sondern es entsteht und wird transformiert in Interaktions- und Kommunikationsprozessen zwischen individuellen und kollektiven Akteuren. Als Handlungskompetenz ist Wissen an Akteure gebunden, enthält Bewertungen und ist gekoppelt an Erfahrungen, mentale Modelle und an Fähigkeiten. Um Wissen ökonomisch in Wert zu setzen, bedarf es der Handlungskompetenz der Akteure, die es kontextspezifisch anwenden und zur Lösung komplexer Probleme zusammenführen (Stehr 1994; Helmstädter 1999; Brödner 2010; Ibert/Kujath 2011; Meusburger/Koch/ Christmann 2011). Durch damit verbundene Lernprozesse verändert sich Wissen dynamisch und vermehrt sich in der Anwendung. Es wird mit Erfahrungswissen, implizitem Wissen und Kontextwissen vergrößert und angereichert. Insofern besitzt es einen kumulativen Charakter.

Arbeits- und organisationswissenschaftliche Studien haben sich vermehrt der Frage gewidmet, wie Wissensarbeit definiert und von „Nicht-Wissensarbeit“" abgegrenzt werden kann. Zwei generische Merkmale scheinen hier von wesentlicher Relevanz: Wissensarbeit ist in hohem Maße durch die Neuartigkeit und hohe Komplexität von Aufgabenstellungen gekennzeichnet (Hube 2005: 33; Kelter/Rief/Bauer et al. 2009), dadurch ist sowohl die Standardisierbarkeit von wissensintensiven Tätigkeiten als auch ihre Leistungsmessung und -steuerung mit den üblichen quantitativen Indikatoren nur in sehr eingeschränktem Maße möglich (Davenport 2010). Die Neuartigkeit bedingt zusammen mit dem nicht wiederkehrenden Charakter von Aufgabenstellungen, die in einem komplexen Umfeld gelöst werden müssen, dass aus der Bewältigung von Aufgaben der Vergangenheit kaum Erkenntnisse über den Aufwand für die Bewältigung zukünftiger Aufgaben abgeleitet werden können. Originäre wissenserzeugende Tätigkeiten sind von hohen Kreativitätsund Lernanforderungen geprägt (Tiemann 2009).

Aufgrund der Einmaligkeit, Komplexität und Kontextabhängigkeit von wissensintensiven Tätigkeiten stellen Teamarbeit und zeitlich befristete Organisationsformen wie die Projektorganisation eine dominante Form der Arbeitsorganisation dar. Komplexe und neuartige Problemlösungen erfordern oft die Integration und Kombination von unterschiedlichen Wissensgebieten und entstehen infolgedessen in komplexen Kommunikations- und Interaktionsprozessen in Teams, Projektgruppen oder Wissensgemeinschaften (,knowledge communities“) (Gibbons/Limoges/Nowotny et al. 1994; Amin/Cohendet 2004; Amin/Roberts 2008; Strambach/Klement 2012), die organisationsinterne und -externe Experten umfassen.

Die relative Unbestimmtheit und Vielfältigkeit dieser geistig-schöpferischen Tätigkeiten in Verbindung mit dem Prozesscharakter der Wissensgenerierung und -verwertung führen dazu, dass dem kreativen Potenzial der Arbeitsplatzinhaber (oder ,Wissensarbeitenden'), ihren Erfahrungen, ihrer Eigenverantwortung und Autonomie im Vergleich zu Nicht-Wissensarbeit ein ungleich höherer Stellenwert zukommt. Originäre Wissensarbeit ist daher durch einen hohen Grad der Subjektivierung gekennzeichnet - im Unterschied zur Objektivierung industrieller Arbeitsorganisation, die darauf ausgerichtet ist, Arbeitsinhalte und -prozesse zu standardisieren und Regeln, Routinen und Vorgehensweisen im Unternehmen zu definieren (Minssen 2012), um damit unabhängig von der ,subjektiven Persönlichkeit' Steuerung und Leistungsmessung zu ermöglichen. Inwieweit die inhärent vorhandenen Handlungsspielräume bei wissensintensiven Tätigkeiten im Sinne der Ziele der jeweiligen Organisation ausgefüllt werden, hängt ganz entscheidend von den individuellen Beschäftigten ab. Wesentlich sind nicht nur fachspezifische Kompetenzen und Erfahrungswissen, sondern auch persönliche Eigenschaften, Einstellungen, Werte und Motivationen.

Aus den Diskursen lässt sich zusammenfassen, dass originäre Wissensarbeit durch die generischen Merkmale Neuartigkeit und Komplexität gekennzeichnet ist, deren unterschiedliche Ausprägung den Grad der zeitlichen Unbestimmtheit und Nichtplanbarkeit dieser geistigen, kreativen Tätigkeiten beeinflussen. Die ökonomische Wertschöpfung der Wissensarbeit wird in erheblichem Maße von der Subjektivität der Wissensarbeitenden bestimmt.

Welche Auswirkungen diese Prozesse auf die physische Mobilität von Wissensarbeitenden haben, ist bislang keineswegs eindeutig geklärt. Obwohl, wie bereits aufgezeigt wurde, empirisch eine Zunahme der physischen Mobilität zu beobachten ist (Schneider/Ruppenthal/Lück et al. 2008: 131 ff.), können gerade immateriell-geistige Tätigkeiten, die nicht an feste Objekte gebunden sind, durch moderne IuK-Technologien häufig auch räumlich unabhängig durchgeführt werden. Der Frage, welche Aussagen sich aufgrund der prägenden Merkmale von Wissensarbeit über die spezifischen Raum-Zeit-Bindungen ableiten lassen und wie diese Merkmale wiederum in Verbindung mit berufsbedingter zirkulärer Mobilität stehen, wird im Folgenden nachgegangen.

\section{Raum-zeitliche Mechanismen von Wissensarbeit}

Mit dem Bedeutungsgewinn der Wissensarbeit in hochentwickelten Ökonomien und Gesellschaften rückt auch die 
Frage nach deren Raum-Zeitlichkeit in den Fokus verschiedener Forschungsdisziplinen. In der arbeits- und industriesoziologischen Forschung werden seit geraumer Zeit Prozesse der zeitlichen und räumlichen Flexibilisierung der Erwerbsarbeit unter dem Schlagwort der „Entgrenzung“ diskutiert. Zeitliche Dimensionen der Entgrenzung betreffen die Dauer der Arbeitszeiten, den Wegfall von festen zeitlichen Vorgaben sowie das Verwischen der klaren Grenzen zwischen Arbeitszeit und Freizeit (Pongratz/Voß 2004; Hinz 2008; Minssen 2012). Auf der räumlichen Seite wird die Auflösung des „Ein-Arbeitsplatz-Modells“ und die Flexibilisierung von Arbeitsorten durch Telearbeit (Gebauer 2002) oder das Arbeiten von Unterwegs (von Streit 2011) diskutiert. Genauere Bezüge zur Wissensarbeit und den zu Grunde liegenden Tätigkeiten werden in diesem Literaturstrang aber kaum hergestellt.

In arbeits- und organisationswissenschaftlichen Studien wird unter dem Stichwort „mobile work“ aufgezeigt, dass komplexere Formen von verteilter Arbeit (,distributed work") und sogenannter ,co-located work“ (räumlich benachbarter Arbeit) entstehen, die mit dem Arbeiten von zuhause und von unterwegs ergänzt werden. Tätigkeiten können generell nach dem Grad ihrer Raum- und Zeitabhängigkeit differenziert werden, je nachdem, ob sie raumabhängig oder -unabhängig bzw. zeitabhängig oder -unabhängig verrichtet werden können. Wiberg (2005) beispielsweise analysiert wissensintensive Dienstleistungstätigkeiten im Telekommunikationsbereich. Er kommt zu dem Schluss, dass lediglich ein kleiner Teil der Tätigkeiten in die Kategorie ,immer und überall“ (,,anytime-anywhere“) fällt und damit sowohl raum- als auch zeitunabhängig ist. Physische Mobilität ist vielfach nicht zu vermeiden, da das Objekt, an dem die wissensintensive Tätigkeit ausgeübt wird, oder die Kunden räumlich verortet sind. Urry (2002: 262 f.), der zwar nicht explizit berufsbezogene Mobilität und Wissensarbeit beleuchtet, weist ebenfalls auf diese Raumbindung hin. Er identifiziert unter anderem „object obligations“ (Ansprüche aus der Sache heraus), welche durch die Verankerung eines Objekts an einem bestimmten Ort eine Ko-Präsenz erfordern, als einen wesentlichen Grund für physische Mobilität.

Hier stellt sich die Frage, welche weiteren Mechanismen räumliche und zeitliche Bindungen von immateriellen wissensintensiven Tätigkeiten bedingen. Neben dieser direkten objektbezogenen Bindung werden indirektere Formen der räumlichen Bindung von Wissen in wirtschaftsgeographischen Forschungen deutlich, welche die Erzeugung und Anwendung von Wissen primär in Bezug zu Innovationsprozessen und weniger aus der Perspektive der Arbeitsteilung untersuchen (Crevoisier/Jeannerat 2009; Cooke/Laurentis/ MacNeill et al. 2010; Gertler 2008; Howells 2012; Jensen/ Johnson/Lorenz et al. 2007; Malecki 2010; Strambach/Halkier 2013). In vielen empirischen Analysen wurde gezeigt, dass räumliche Nähe den Austausch und die Entstehung von
Wissen in Innovationsprozessen befördert (Asheim/Gertler 2005; Tödtling/Lehner/Trippl 2006; Lagendijk/Lorentzen 2007; Martin/Moodysson 2011). Persönliche Kontakte (,face to face") werden als wesentlich für den Aufbau von Vertrauen und die Übertragung von implizitem Erfahrungswissen betrachtet. Lokalisierte Lernprozesse der Akteure über die Zeit führen zu lokalisierten Wissensbeständen an bestimmten Standorten, die wiederum die Notwendigkeit generieren, zumindest temporär vor Ort zu sein, um an lokalisierten Kommunikationsprozessen (dem , local buzz') teilzuhaben, da Übertragungseffekte von Wissen (spillover) nur begrenzte räumliche Reichweiten haben (Gertler 2003; Bathelt/Malmberg/Maskell 2004; Malmberg/Maskell 2006; Torre 2008).

Neben der räumlichen Nähe kommt auch nichtgeographischen Formen von Nähe und Distanz eine wichtige Funktion zu. Nichtgeographische Formen von Nähe und Distanz werden durch kognitive, organisationale, institutionelle, soziale oder kulturelle Unterschiede zwischen den Akteuren charakterisiert (Boschma 2005; Knoben/Oerlemans 2006; Hansen 2014). Diese nicht geographischen, sondern relationalen Distanzen (Ibert 2010) wirken sich immer dann aus, wenn Wissensarbeit in Kollaboration erfolgt. Durch die Personengebundenheit von Wissen und den Umstand, dass Wissen in Interaktions- und Kommunikationsprozessen entsteht, erschweren insbesondere große kognitive Distanzen zwischen den Akteuren den Wissensaustausch, die Absorptionsfähigkeit von Wissen und die Entwicklung eines gemeinsamen Verständnisses für komplexe Problemstellungen (Cohen/Levinthal 1990; Nooteboom 2010). Aufgrund der vielfältigen Kommunikationskanäle, die in Face-to-Face-Kontakten genutzt werden können, ist die Herstellung von temporärer räumlicher Nähe oft erforderlich, um in komplexen Interaktions- und Kommunikationsprozessen kognitive Nähe und eine ,gemeinsame Sprache herzustellen. Diese wiederum erleichtert Lernprozesse und das Finden von kreativen Lösungen in der Zusammenarbeit. Nichtgeographische relationale Distanzen stehen mit der physischen bzw. geographischen Distanz und dem materiellen Kontext in enger und komplexer Verbindung (Ibert 2011; Kujath/Stein 2011; Hansen 2014) und beeinflussen die raum-zeitliche Bindung wissensintensiver Tätigkeiten und Praktiken.

Ibert/Thiel (2009: 212 f.) stoßen im Rahmen einer zeitgeographischen Debatte an, dass wissensintensive Arbeit anderen raum-zeitlichen Mechanismen folgt als klassische Erwerbsarbeit. Aufbauend auf den Erkenntnissen zu Nähe und Distanz in Wissensaustausch- und Innovationsprozessen argumentieren sie, dass Wissensarbeit durch eine höhere Dynamik, Flüchtigkeit und Desintegration gekennzeichnet ist als z. B. materielle Tätigkeiten im Kontext einer tayloristischen Arbeitsorganisation. Zur Beschreibung von wissensintensiver Arbeit nennen sie die fünf Attribute „kollaborativ“, 
„informell“, „situiert“, „mobil“ und „virtuell“, welche weitere Hinweise auf die raum-zeitlichen Besonderheiten von wissensintensiver Arbeit geben. Mit den Attributen „mobil“ und ,virtuell“ deutet sich an, dass Mobilität eine wesentliche Determinante wissensintensiver Tätigkeiten ist. So wird die Zunahme physischer Mobilität durch räumlich verteilte Wissensexpertisen erklärt, die Reisen oftmals unumgänglich machen. Weniger substituierend, sondern eher ergänzend wirken neue IuK-Technologien, durch die ein großer Teil der notwendigen Interaktion auch virtuell abgewickelt wird. In Bezug auf raum-zeitliche Bindung wird festgestellt, dass wissensintensive Tätigkeiten keiner klaren Fixierung unterliegen, sondern weniger raum-zeitlich gebunden sind. Sie sind aufgrund der Wichtigkeit von Kollaboration und Interaktion allerdings nicht als losgelöst zu bezeichnen, sondern als situiert (Ibert/Thiel 2009: 210 f.). Neben dem „Mehr“ an Mobilität weisen diese Arbeiten daher implizit auf eine Dynamik und Flexibilisierung von Mobilität hin. Tätigkeiten und Arbeitsschritte erfordern aufgrund ihrer Reflexivität und Unvorhersehbarkeit ein dynamisches Wechselverhältnis von Ort- und Zeitgebundenheit bzw. -ungebundenheit. Welche genauen raum-zeitlichen Mechanismen den einzelnen Tätigkeiten der Wissensarbeit zu Grunde liegen, ist allerdings noch eine weitgehend offene Frage.

Aufgrund des geführten Diskurses darf angenommen werden, dass räumliche Nähe und Face-to-Face-Kontakte vorrangig immer dann hergestellt werden, wenn die Komplexität und Neuartigkeit der Tätigkeiten und Arbeitsschritte sehr hoch sind und komplexe Aufgaben bewältigt werden müssen, deren Problemlösung in Kollaboration mit anderen Akteuren, sei es organisationsintern oder -extern, erleichtert wird. Die jeweilige Ausprägung der Neuartigkeit und Komplexität der immateriellen Tätigkeiten beeinflusst die zeitliche Unbestimmtheit und Nichtplanbarkeit der Wissensarbeit. Wie diese ausgeführt wird, an welchem Ort und zu welcher Zeit und in Abstimmung mit welchen Kollaborationspartnern, hängt von den jeweiligen beteiligten Akteuren und deren nichtgeographischen Nähe- und Distanzrelationen ab. Die temporäre räumliche Nähe wiederum verändert die nichtgeographischen Nähe- und Distanzrelationen zwischen den Akteuren, die im Prozess der gemeinsamen Wissensproduktion nicht statisch sind.

Die Notwendigkeit der Kollaboration in der Wissensarbeit bedingt nicht zwangsläufig die räumliche Bindung von Tätigkeiten und somit physische Mobilität. Informations- und Kommunikationstechnologien können die Raumbindung von weniger komplexen wissensintensiven Tätigkeiten ergänzen, wenn auch zeitliche Bindungen für die Abstimmungsprozesse in der Zusammenarbeit bestehen bleiben. Sogenannte Sekundäraufgaben mit niedriger Komplexität, wie Dokumentation, Organisations- oder wenig komplexe Kommunikationsarbeit, können durch die Nutzung von IuK-Technologien unabhängig von Raum und
Zeit bearbeitet werden. Sie weisen geringe räumliche und zeitliche Bindungen auf und lassen sich z. B. ,anywhere and anytime“ (Wiberg 2005) oder von unterwegs ,working while mobile" (Cohen 2010) erledigen.

Daneben kann kreative geistige Arbeit mit hoher Komplexität auch in Einzelarbeit ausgeführt werden. Vor allem, wenn es um das Entwickeln neuer Ideen, das Schreiben von komplexen Texten etc. geht, ist individuelle konzentrierte Arbeit oftmals unerlässlich. Diesen Phänomenen ist aber bislang aus sozialwissenschaftlicher Sicht kaum Aufmerksamkeit geschenkt worden. Helbrecht (2004) merkt an, dass „Denken“ prinzipiell überall möglich ist. Dennoch darf vermutet werden, dass diese Tätigkeiten zwar geringe zeitliche Bindungen, aber durchaus indirekte Raumbindungen aufweisen, da sie oftmals ein spezifisches räumliches Umfeld benötigen. Wie allerdings solche „Denkräume“ für die Erbringung geistig-kreativer Einzelarbeiten beschaffen sein müssen, welches Umfeld und welche Atmosphäre förderlich sind, ist vermutlich zu einem hohen Grad subjektiv und individuell.

Abschließend kann festgehalten werden, dass es trotz zunehmender Entgrenzung von Arbeit, unterstützt durch IuK-Technologien, dennoch spezifische Situationen der Wissensarbeit gibt, in denen entweder Raumbindung oder Zeitbindung oder beides erforderlich ist. Bedingt durch die Anteile und das Wechselverhältnis von komplexen, neuartigen Aufgaben und Routinetätigkeiten, Kollaboration und Einzelarbeit sowie der Nichtplanbarkeit und Unvorhersehbarkeit stellt Wissensarbeit hohe räumliche und zeitliche Flexibilitätsanforderungen. Raum-zeitliche Konstellationen von Wissensarbeit sind nicht statisch, sondern verändern sich dynamisch, je nach Anforderung im Arbeitsprozess und in Abhängigkeit von den jeweiligen Akteurskonstellationen. Dadurch bilden sich komplexe, nur schwierig vorhersehbare und planbare Dynamiken von raum-zeitlich abhängigen und unabhängigen Tätigkeiten heraus. Aufgrund der beschriebenen zu Grunde liegenden Prozesse der Wissensgenerierung ist dabei davon auszugehen, dass diese Dynamiken Wissensarbeitende mit höheren Anforderungen an Wissensintensität und Kollaboration besonders betreffen.

\section{Implikationen für die berufsbedingte zirkuläre Mobilität}

Die Zusammenführung der diskutierten Erkenntnisse aus beiden Forschungssträngen verdeutlicht, dass der Wandel der berufsbedingten zirkulären Mobilität neben dem bereits empirisch sichtbaren quantitativen Zuwachs auch eine qualitative Komponente beinhaltet. Ableiten lässt sich, dass Wissensarbeitende nicht nur mehr mobil sind, sondern wissensintensive Tätigkeiten auch mit dynamischen und flexiblen Anforderungen an die berufsbedingte zirkuläre 
Mobilität in Verbindung stehen, über deren Ausprägung bislang noch wenig gesicherte Erkenntnisse vorhanden sind. Die Dynamisierung und Flexibilisierung scheint sich in verschiedenen raum-zeitlichen Dimensionen zu vollziehen, die in enger, wechselseitiger Beziehung stehen und sich gegenseitig beeinflussen (siehe Abb. 1). Aus analytischen Gründen werden sie im Folgenden getrennt voneinander dargestellt.

Räumlich zeichnet sich berufsbedingte zirkuläre Mobilität im Kontext wissensintensiver Arbeit durch Multiskalarität (1) aus. Hierauf verweisen beide Forschungsstränge. Dienst- und Geschäftsreisen, aber auch berufsbedingte Pendelbewegungen vollziehen sich über verschiedene räumliche Skalen, von der lokalen bis hin zur globalen Maßstabsebene. Bedingt durch fortschreitende Fragmentierung von Wertschöpfungsketten und das Offshoring von wissensintensiven Tätigkeiten erfordern komplexe und neuartige Probleme oft die Kombination und Integration von spezialisierten Wissensbasen heterogener Akteure, die in unterschiedlichen Kontexten und auf unterschiedlichen räumlichen Skalen situiert sind. Die Prozess- und Kontextabhängigkeit von Wissensgenerierung und -transformation, die Akteurskonstellationen und der Grad der Kollaboration wirken sich je nach Erfordernissen im Arbeitsprozess auf die Multiskalarität räumlicher Mobilität aus.

Multilokalität (2): Multilokale Wohnarrangements sind eine bevorzugte Organisationsform von Wissensarbeitenden und beeinflussen die räumliche Mobilität zwischen wiederkehrenden Orten. Wissensintensive und kreative Arbeitsprozesse sind aufgrund der Notwendigkeit von Interaktion und räumlicher Ko-Präsenz, aber auch aufgrund der Einzeltätigkeiten, die ein besonderes Umfeld erfordern, geprägt

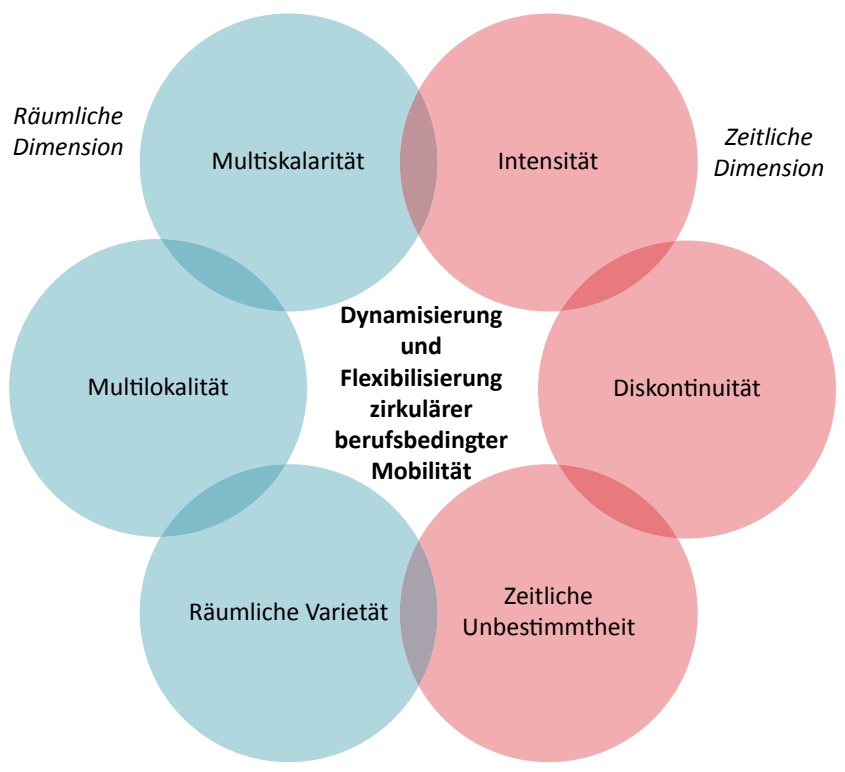

Abb. 1 Dimensionen der Dynamisierung und Flexibilisierung zirkulärer berufsbedingter Mobilität. (Quelle: Eigene Darstellung) durch Mehrörtlichkeit. In Verbindung mit der Subjektivierung, Eigenverantwortung und Autonomie, die Wissensarbeit im Vergleich zu Nicht-Wissensarbeit auszeichnet, bestehen flexible Handlungsspielräume der subjektiven Organisation und Synchronisation der räumlichen berufsbedingten Mobilität.

Räumliche Varietät (3): Die Raumkonstellationen in der Wissensproduktion können durch wiederkehrende Orte oder durch einmalige und neuartige Orte geprägt sein. Durch zeitlich befristete Projekte mit wechselnden beteiligten Akteuren werden im Arbeitsprozess auch vermehrt einmalige oder neuartige Orte aufgesucht, welche nur vorübergehend eine Rolle spielen und danach von anderen abgelöst werden. Insbesondere wissensintensive Tätigkeiten und Interaktionsprozesse zwischen Akteuren, die nicht objektgebunden sind, können an unterschiedlichen Orten stattfinden und erhöhen damit die räumliche Varietät der zirkulären Mobilität. Zudem kann die dynamische Verschiebung der relationalen Näheund Distanzdimensionen in den Interaktionsprozessen der Wissensgenerierung und -transformation die Notwendigkeiten dynamisch verändern, inwieweit räumliche Nähe herzustellen ist und damit Orte wiederkehrend aufzusuchen sind.

Zeitliche Intensität (4): Die Mobilitätsforschung unterstreicht, dass die räumliche Mobilität von Wissensarbeitenden durch zeitliche Intensität geprägt ist. Dienstreisen und geschäftliche Wege sind durch eine zeitliche Verdichtung gekennzeichnet, die nicht zuletzt durch die Notwendigkeit der Interaktion mit unternehmensinternen und -externen Akteuren im Arbeitsprozess entsteht. Die Projektorganisation als primäre Organisationsform von Wissensarbeit bedingt die Synchronisation und Koordination von unterschiedlichen Projekten, in die Wissensarbeitende involviert sind. Die darauf zurückzuführende größere zeitliche Intensität der Arbeit wirkt sich wiederum auch auf andere berufsbedingte zirkuläre Wege wie das tägliche Pendeln aus, die entsprechend koordiniert und angepasst werden müssen.

Diskontinuität (5): Durch die Prozessorientierung und Situiertheit einzelner Arbeitsschritte lockern sich zeitlich regelmäßige Muster und Routinen. Dies betrifft sowohl tages- und wochenzeitliche als auch längerfristige Rhythmen. So kann beispielsweise der tägliche Weg zwischen Wohnung und Arbeit aufgrund der temporären Projektorganisation und wechselnden Arbeitsanforderungen, Kollaborations- und Abstimmungserfordernissen weniger im Rahmen von einheitlichen Tagesrhythmen zurückgelegt werden. Sekundäraufgaben und kreative Einzelarbeiten können zudem an Orten außerhalb des Arbeitsplatzes verrichtet werden, dies begünstigt eine weitere Auflösung regelmäßiger zeitlicher Rhythmen. Häufige und verdichtete Dienstreiseaktivität und geschäftliche Wege tragen zusätzlich zu einer Erosion regelmäßiger tages-, wochenzeitlicher und auch längerfristiger Rhythmen bei. 
Zeitliche Unbestimmtheit (6): Aufgrund der Reflexivität, des Prozesscharakters und der Kontextabhängigkeit von wissensintensiven Tätigkeiten sowie durch die im Arbeitsprozess auftretenden und rasch veränderlichen Erfordernisse des Wissensaustauschs und der Wissenstransformation kann räumliche Mobilität schwieriger geplant und vorbereitet werden. Berufsbedingte zirkuläre räumliche Bewegungen treten vermehrt situativ und spontan auf. Die Heterogenität der Akteurskonstellationen sowie deren räumliche Situiertheit in Verbindung mit der Komplexität und Neuartigkeit der jeweiligen Wissensproduktion beeinflussen die zeitliche Synchronisation und sind somit nur zum Teil selbstbestimmt.

Festhalten lässt sich, dass der Kern von Wissensarbeit aus wissensintensiven Tätigkeiten besteht, die durch Eigenschaften der Komplexität, Neuartigkeit und Kollaboration gekennzeichnet sind. Die verschiedenen raum-zeitlichen Dimensionen, die in enger, wechselseitiger Beziehung stehen und sich gegenseitig beeinflussen, lassen auf eine Dynamisierung und Flexibilisierung von berufsbedingter zirkulärer Mobilität schließen, die mit Wissensarbeit in Verbindung stehen.

\section{Fazit und Ausblick}

Dieser Beitrag hatte zum Ziel, durch die Zusammenführung der konzeptionell weitgehend getrennten Stränge der interdisziplinären Mobilitätsforschung und der raumbezogenen Innovations- und Wissensforschung Synergiepotenziale aufzuzeigen, die genutzt werden können, um differenzierter zu erforschen, wie sich Wissensarbeit auf die berufsbedingte zirkuläre Mobilität auswirkt. Dabei wurden generelle Mechanismen der raum-zeitlichen Bindung von Wissensarbeit und ihre Auswirkungen auf berufsbedingte zirkuläre Mobilität herausgearbeitet.

Aus den theoretisch-konzeptionellen Erkenntnissen lässt sich ableiten, dass wissensintensive Tätigkeiten, die den Kern von Wissensarbeit darstellen, im Vergleich zu materiellen Tätigkeiten oder Routineaufgaben mit fluiden Bindungen an Raum und Zeit einhergehen und damit als situiert bezeichnet werden können. Bedingt durch die Ausprägungen der Tätigkeiten und ihrer Wissensintensität, durch das Wechselverhältnis von komplexen, neuartigen Aufgaben und Routinetätigkeiten und von Kollaboration und Einzelarbeit bilden sich dynamische und flexible raum-zeitliche Mobilitätsmuster heraus. Dieser Wandel kann auf der räumlichen Seite anhand der drei Dimensionen Multiskalarität, Multilokalität und der räumlichen Varietät näher beschrieben werden, während sich die zeitlichen Dimensionen durch Intensität, Diskontinuität und zeitliche Unbestimmtheit fassen lassen. Die genauen Zusammenhänge zwischen einzelnen Tätigkeiten, auch vor dem Hintergrund von branchen- und unternehmensspezifischen Kontexten, und den
Auswirkungen auf berufsbedingte zirkuläre Mobilität sind aber noch nicht gut untersucht.

Zukünftige empirische Forschungen, welche die dargelegten Zusammenhänge zwischen wissensintensiven Tätigkeiten und Mobilitätsdynamiken systematisch untersuchen, können hier zur weiteren Fundierung beitragen. Um die fluiden Dynamiken berufsbedingter zirkulärer Mobilität zu fassen, gilt es auch, neben der Kombination von erprobten quantitativen und qualitativen Instrumenten neue Methoden und Wege der Datengewinnung zu erschließen, beispielsweise durch den Einsatz von sogenannten ,mobile methods" (mobilen Erhebungsmethoden). Eine weitere Herausforderung wird es sein, die aufgezeigten Mobilitätsdynamiken auch in planerische Handlungskontexte stärker zu integrieren. Es ist davon auszugehen, dass sich der sozioökonomische Strukturwandel zur Wissensökonomie in hochentwickelten Ländern wie Deutschland weiter fortsetzt und damit zukünftig eine wachsende Anzahl von Erwerbstätigen mit flexibleren Mobilitätsanforderungen konfrontiert sein wird. Die Problematik, einerseits die besonderen dynamischen und individualisierten beruflichen Mobilitätserfordernisse zu berücksichtigen und auf der anderen Seite eine umweltgerechte, ökonomische und soziale Gestaltung zu gewährleisten, erfordert innovative Ansätze für die Verkehrsplanung und das Mobilitätsmanagement.

Für die wertvollen Hinweise und Anregungen der beiden anonymen Gutachter und der Schriftleitung möchten wir uns an dieser Stelle sehr herzlich bedanken.

\section{Literatur}

Amin, A.; Cohendet, P. (2004): Architectures of Knowledge: Firms, Capabilities, and Communities. Oxford.

Amin, A.; Roberts, J. (Hrsg.) (2008): Community, Economic Creativity, and Organization. Oxford.

Antonelli, C. (2005): Models of Knowledge and Systems of Governance. In: Journal of Institutional Economics 1, 1, 51-73.

Asheim, B.; Gertler, M. S. (2005): The Geography of Innovation: Regional Innovation Systems. In: Fagerberg, J.; Mowery, D. C.; Nelson, R. R. (Hrsg.): The Oxford Handbook of Innovation. New York, 291-317.

Bathelt, H.; Malmberg, A.; Maskell, P. (2004): Clusters and Knowledge: Local Buzz, Global Pipelines and the Process of Knowledge Creation. In: Progress in Human Geography 28, 1, 31-56.

Beaverstock, J. (2002): Transnational elites in global cities: British expatriates in Singapore's financial district. In: Geoforum 33, 4, $525-538$

Bonnet, E.; Orain, R. (2010): Job Careers and Job Mobility. In: Schneider, N. F; Collet, B. (Hrsg.): Mobile Living Across Europe II. Causes and Consequences of Job-Related Spatial Mobility in Cross-National Comparison. Opladen, Farmington Hills, 289-315.

Boschma, R. (2005): Proximity and Innovation: A Critical Assessment. In: Regional Studies 39, 1, 61-74.

Brödner, P. (2010): Wissensteilung und Wissenstransformation. In: Moldaschl, M.; Stehr, N. (Hrsg.): Wissensökonomie und Innovation: Beiträge zur Ökonomie der Wissensgesellschaft. Marburg, 455-480. 
Cohen, R. L. (2010): Rethinking ,mobile work': boundaries of space, time and social relation in the working lives of mobile hairstylists. In: Work, Employment and Society 24, 1, 65-84.

Cohen, W. M.; Levinthal, D. M. (1990): Absorptive Capacity: A New Perspective on Learning and Innovation. In: Administrative Science Quarterly 35, 1, 128-152.

Cooke, P.; Laurentis, C.; MacNeill, S.; Collinge, C. (Hrsg.) (2010): Platforms of Innovation: Dynamics of new Industrial Knowledge Flows. Cheltenham, Northampton.

Crevoisier, O.; Jeannerat, H. (2009): Territorial Knowledge Dynamics. From the Proximity Paradigm to Multi-location Milieus. In: European Planning Studies 17, 8, 1223-1241.

Davenport, T. H. (2010): Process Management for Knowledge Work. In: Vom Brocke, J.; Rosemann, M. (Hrsg.): Handbook on Business Process Management 1: Introduction, Methods, and Information Systems. Berlin, Heidelberg, 17-35.

Davidson, R.; Cope, B. (2003): Business Travel. Conferences, Incentive Travel, Corporate Hospitality and Corporate Travel. Essex.

Didero, M.; Pfaffenbach, C. (2014): Multilokalität und Translokalität. Konzepte und Perspektiven eines Forschungsfelds. In: Geographische Rundschau 66, 11, 4-9.

Dittrich-Wesbuer, A.; Kramer, C. (2014): Heute hier - morgen dort. In: Geographische Rundschau 66, 11, 46-52.

Dolfsma, W.; Soete, L. (Hrsg.) (2006): Understanding the Dynamics of a Knowledge Economy. Cheltenham, Northampton.

Einig, K.; Pütz, T. (2007): Regionale Dynamik der Pendlergesellschaft. Entwicklung von Verflechtungsmustern und Pendlerdistanzen. In: Informationen zur Raumentwicklung 2/3 (2007), 73-91.

Foray, D. (2004): Economics of Knowledge. Cambridge, Mass. (u. a).

Gather, M.; Kagermeier, A.; Lanzendorf, M. (2008): Geographische Mobilitäts- und Verkehrsforschung. Berlin, Stuttgart.

Gebauer, I. (2002): Die Auswirkungen häuslicher Telearbeit auf das Verkehrsverhalten und Aktionsräume: eine Sekundäranalyse als explorative Studie. Stuttgart. = Diskussionsbeiträge, Institut für Geographie, Stuttgart, Band 12.

Gertler, M. S. (2003): Tacit Knowledge and the Geography of Context, or the Undefinable Tacitness of Being (There). In: Journal of Economic Geography 3, 1, 75-99.

Gertler, M. S. (2008): Buzz Without Being There? Communities of Practice in Context. In: Amin, A.; Roberts, J. (Hrsg.): Community, Economic Creativity, and Organization. Oxford, 203-226.

Gibbons, M.; Limoges, C.; Nowotny, H.; Schwartzman, S.; Scott, P. (1994): The New Production of Knowledge: The Dynamics of Science and Research in Contemporary Societies. London.

Giza-Poleszczuk, A.; Stec, M.; Komendant, A.; Rüger, H. (2010): Social Class and Job Mobilities. Human Capital, External Constraints and Mobility Framing. In: Schneider, N. F.; Collet, B. (Hrsg.): Mobile Living Across Europe II. Causes and Consequences of JobRelated Spatial Mobility in Cross-National Comparison. Opladen, Farmington Hills, 194-213.

Gornig, M.; Mundelius, M. (2012): Reurbanisierung und wissensbasierte Ökonomie. In: Brake, K.; Herfert, G. (Hrsg.): Reurbanisierung. Materialität und Diskurs in Deutschland. Wiesbaden, $130-150$.

Haas, A.; Hamann, S. (2008): Pendeln - ein zunehmender Trend, vor allem bei Hochqualifizierten. In: IAB-Kurzberichte 2008, 6. http:// doku.iab.de/kurzber/2008/kb0608.pdf (21.06.2015).

Hall, A. (2007): Tätigkeiten und berufliche Anforderungen in wissensintensiven Berufen. http://www.bmbf.de/pubRD/sdi-03-07.pdf (18.03.2014).

Hansen, T. (2014): Substitution or Overlap? The Relations between Geographical and Non-spatial Proximity Dimensions in Collaborative Innovation Projects. In: Regional Studies. http://www.tandfonline.com/doi/pdf/10.1080/00343404.2013.873120 (23.3.2014).

Heineberg, H. (2007): Einführung in die Anthropogeographie/Humangeographie. Paderborn, München.
Helbrecht, I. (2004): Denkraum Stadt. In: Siebel, W. (Hrsg.): Die europäische Stadt. Frankfurt a. M., 422-432.

Helmstädter, E. (1999): Arbeitsteilung und Wissensteilung: ihre institutionenökonomische Begründung. In: Brödner, P.; Helmstädter, E.; Widmaier, B. (Hrsg.): Wissensteilung: Zur Dynamik von Innovation und kollektivem Lernen. München, 33-54.

Hinz, S. (2008): Erwerbstätigkeit im Wandel unter besonderer Berücksichtigung der Frauenerwerbsarbeit und der Vereinbarkeit von Beruf und Familie. Tönning (u. a.).

Howells, J. (2012): The geography of knowledge: never so close but never so far apart. In: Journal of Economic Geography 12, 5, $1003-1020$

Hube, G. (2005): Beitrag zur Analyse und Beschreibung von Wissensarbeit. Heimsheim.

Ibert, O. (2010): Relational Distance: Sociocultural and Time - Spatial Tensions in Innovation Practices. In: Environment and Planning A $42,1,187-204$.

Ibert, O. (2011): Dynamische Geographien der Wissensproduktion Die Bedeutung physischer wie relationaler Distanzen in interaktiven Lernprozessen. In: Ibert, O.; Kujath, H. J. (Hrsg.): Räume der Wissensarbeit: Zur Funktion von Nähe und Distanz in der Wissensökonomie. Wiesbaden, 49-69.

Ibert, O.; Kujath, H. J. (2011): Wissensarbeit aus räumlicher Perspektive - Begriffliche Grundlagen und Neuausrichtungen im Diskurs. In: Ibert, O.; Kujath, H. J. (Hrsg.): Räume der Wissensarbeit. Zur Funktion von Nähe und Distanz in der Wissensökonomie. Wiesbaden, 9-48.

Ibert, O.; Thiel, J. (2009): Situierte Analyse, dynamische Räumlichkeiten. Ausgangspunkte, Perspektiven und Potenziale einer Zeitgeographie der wissensbasierten Ökonomie. In: Zeitschrift für Wirtschaftsgeographie 53, 4, 209-223.

Jensen, M. B.; Johnson, B.; Lorenz, E.; Lundvall, B. A. (2007): Forms of knowledge and modes of innovations. In: Research Policy 36, $5,680-693$

Kelter, J.; Rief, S.; Bauer, W.; Haner, U.-E. (2009): Office 21-Studie: Information Work. Über die Potenziale von Informationsund Kommunikationstechnologien bei Büro- und Wissensarbeit. http://www.office21.de/content/dam/office21/de/documents/Publikationen/FraunhoferIAO-Studie Information Work2009.pdf (21.2.2014).

Kesselring, S.; Vogl, G. (2010a): Die Mobilisierung der Arbeitskraft. Zur Normalisierung, Rationalisierung und Verdichtung von Dienstreisen. In: Götz, I.; Lemberger, B.; Lehnert, K.; Schondelmayer, S. (Hrsg.): Mobilität und Mobilisierung. Arbeit im sozioökonomischen, politischen und kulturellen Wandel. Frankfurt a. M., New York, 45-60. = Schriftenreihe Arbeit und Alltag, Band 1.

Kesselring, S.; Vogl, G. (2010b): Betriebliche Mobilitätsregime. Die sozialen Kosten mobiler Arbeit. Berlin.

Knoben, J.; Oerlemans, L. A. G. (2006): Proximity and inter-organizational collaboration: A literature review. In: International Journal of Management Reviews 8, 2, 71-89.

Kohl, H. (2014): Zum Wandel berufsbedingter Mobilität in der Wissensökonomie. In: Geographische Rundschau 66, 12, 26-31.

Kujath, H. J. (2010): Institutionen und räumliche Organisation der Wissensökonomie. In: Kujath, H. J.; Zillmer, S. (Hrsg.): Räume der Wissensökonomie. Implikationen für das deutsche Städtesystem. Berlin, 51-81. = Stadt- und Regionalwissenschaften, Band 6 .

Kujath, H. J.; Stein, A. (2011): Lokale Wissenskonzentration in den globalen Beziehungsräumen der Wissensökonomie. In: Ibert, O.; Kujath, H. J. (Hrsg.): Räume der Wissensarbeit. Zur Funktion von Nähe und Distanz in der Wissensökonomie. Wiesbaden, 127-154.

Lagendijk, A.; Lorentzen, A. (2007): Proximity, Knowledge and Innovation in Peripheral Regions. On the Intersection between Geographical and Organizational Proximity. In: European Planning Studies $15,4,457-466$. 
Lanzendorf, M.; Scheiner, J. (2004): Verkehrsgenese als Herausforderung für Transdisziplinarität. Stand und Perspektiven der Forschung. In: Dalkmann, H.; Lanzendorf, M.; Scheiner, J. (Hrsg.): Verkehrsgenese. Entstehung von Verkehr sowie Potenziale und Grenzen der Gestaltung einer nachhaltigen Mobilität. Mannheim, 11-38. = Studien zur Mobilitäts- und Verkehrsforschung, Band 5.

Larsen, J.; Axhausen, K. W.; Urry, J. (2006): Geographies of Social Networks: Meeting, Travel and Communications. In: Mobilities $1,2,161-283$.

Limmer, R. (2005): Berufsmobilität und Familie in Deutschland. In: Zeitschrift für Familienforschung 17, 2, 96-114.

Limmer, R.; Schneider, N. F. (2008): Studying Job-related Spatial Mobility in Europe. In: Schneider, N. F.; Meil, G. (Hrsg.): Mobile Living Across Europe I. Relevance and Diversity of Job-Related Spatial Mobility in Six European Countries. Opladen, Farmington Hills, 13-46.

Mahroum, S. (2000): Highly skilled globetrotters: mapping the international migration of human capital. In: R \& D Management 30, $1,23-31$.

Malecki, E. J. (2010): Everywhere? The Geography of Knowledge. In: Journal of Regional Science 50, 1, 493-513.

Malmberg, A.; Maskell, P. (2006): Localized Learning Revisited. In: Growth and Change 37, 1, 1-18.

Martin, R.; Moodysson, J. (2011): Innovation in Symbolic Industries: The Geography and Organization of Knowledge Sourcing. In: European Planning Studies 19, 7, 1183-1203.

Meusburger, P.; Koch, G.; Christmann, G. B. (2011): Nähe- und Distanz-Praktiken in der Wissenserzeugung - Zur Notwendigkeit einer kontextbezogenen Analyse. In: Ibert, O.; Kujath, H. J. (Hrsg.): Räume der Wissensarbeit. Zur Funktion von Nähe und Distanz in der Wissensökonomie. Wiesbaden, 221-249.

Minssen, H. (2012): Arbeit in der modernen Gesellschaft. Eine Einführung. Wiesbaden.

Moldaschl, M. (2012): Mythen der Modernisierung - Arbeit in der Wissensökonomie. In: Priddat, B. P.; West, K.-W. (Hrsg.): Modernität der Industrie. Marburg, 215-248.

Moldaschl, M.; Stehr, N. (2010): Eine kurze Geschichte der Wissensökonomie. In: Moldaschl, M.; Stehr, N. (Hrsg.): Wissensökonomie und Innovation. Beiträge zur Ökonomie der Wissensgesellschaft. Marburg, 9-76.

Nooteboom, B. (2010): A Cognitive Theory of the Firm: Learning, Governance and Dynamic Capabilities. Cheltenham.

Ohnmacht, T. (2006): Mapping Social Networks in Space and Time. Arbeitsberichte Verkehr und Raumplanung, 341, IVT, ETH Zürich. http://e-collection.library.ethz.ch/eserv/eth:28901/eth-2890101.pdf (20.06.2015).

Polanyi, M. (1985): Implizites Wissen. Frankfurt a. M.

Pongratz, H.; Voß, G. G. (2004): Arbeitskraftunternehmer: Erwerbsorientierungen in entgrenzten Arbeitsformen. Berlin.

PTV AG; Fell, B.; Schönfelder, S.; Axhausen, K. W. (2000): Mobidrive questionnaires. In: Arbeitsberichte Verkehrs- und Raumplanung, 52. http://e-collection.library.ethz.ch/eserv/eth:24342/ eth-24342-01.pdf (21.06.2015).

Rüger, H.; Feldhaus, M.; Becker, K. S.; Schlegel, M. (2011): Zirkuläre berufsbezogene Mobilität in Deutschland: Vergleichende Analysen mit zwei repräsentativen Surveys zu Formen, Verbreitung und Relevanz im Kontext der Partnerschafts- und Familienentwicklung. In: Comparative Population Studies - Zeitschrift für Bevö1kerungswissenschaft 36, 1, 193-220.

Scheiner, J. (2009): Sozialer Wandel, Raum und Mobilität. Empirische Untersuchungen zur Subjektivierung der Verkehrsnachfrage. Wiesbaden.

Scheiner, J.; Blotevogel, H.-H.; Frank, S.; Holz-Rau, C.; Schuster, N. (2013): Mobilitäten und Immobilitäten: Menschen - Ideen - Dinge - Kulturen - Kapital. Eine Einleitung. In: Scheiner, J.; Blotevogel, H.-H.; Frank, S.; Holz-Rau, C.; Schuster, N. (Hrsg.):
Mobilitäten und Immobilitäten: Menschen - Ideen - Dinge - Kulturen - Kapital. Essen, 9-18. = Dortmunder Beiträge zur Raumplanung, Band 142.

Schliephake, K.; Schenk, T. (2005): Verkehr und Mobilität. In: Schenk, T.; Schliephake, K. (Hrsg.): Allgemeine Anthropogeographie. Gotha, 531-580.

Schneider, N. F.; Meil, G. (Hrsg.) (2008): Mobile Living Across Europe I. Relevance and Diversity of Job-Related Spatial Mobility in Six European Countries. Opladen, Farmington Hills.

Schneider, N. F.; Ruppenthal, S.; Lück, D.; Rüger, H.; Dauber, A. (2008): Germany - A Country of Locally Attached but Highly Mobile People. In: Schneider, N. F.; Meil, G. (Hrsg.): Mobile Living Across Europe I. Relevance and Diversity of Job-Related Spatial Mobility in Six European Countries. Opladen, Farmington Hills, 105-147.

Schneider, N. F.; Rüger, H.; Münster, E. (2009): Berufsbedingte räumliche Mobilität in Deutschland. Formen, Verbreitung und Folgen für Gesundheit, Wohlbefinden und Familienleben. In: Arbeitsmedizin, Sozialmedizin, Umweltmedizin 44, 7, 400-409.

Schöller-Schwedes, O.; Rammler, S. (2008): Mobile Cities. Dynamiken weltweiter Stadt- und Verkehrsentwicklung. Berlin.

Siedentop, S. (2012): Die Rückkehr der Städte? Zum aktuellen Stand der Reurbanisierungsdebatte. In: Gans, P.; Westerheide, P. (Hrsg.): Zurück in die Stadt?! Mannheim, 3-18. = Mannheimer Schriften zu Wohnungswesen, Kreditwirtschaft und Raumplanung, Band 10.

Stehr, N. (1994): Arbeit, Eigentum und Wissen: Zur Theorie von Wissensgesellschaften. Frankfurt a. M.

Strambach, S. (2011): Herausforderungen der Wissensökonomie. Strukturen, Prozesse und neue Dynamiken im globalen Strukturwandel. In: RegioPol - Zeitschrift für Regionalwirtschaft 1/2, (2011), 25-33.

Strambach, S.; Halkier, H. (2013): Reconceptualizing Change - Path Dependency, Path Plasticity and Knowledge Combination. In: Zeitschrift für Wirtschaftsgeographie 57, 1-2, 1-14.

Strambach, S.; Klement, B. (2012): Cumulative and Combinatorial Micro-dynamics of Knowledge: The Role of Space and Place in Knowledge Integration. In: European Planning Studies 20, 11, $1843-1866$.

von Streit, A. (2011): Entgrenzter Alltag - Arbeiten ohne Grenzen? Das Internet und die raum-zeitlichen Organisationsstrategien von Wissensarbeitern. Bielefeld.

Tiemann, M. (2009): Wissensintensive Berufe. http://www.bibb.de/ dokumente/pdf/a22_preprint02_Tiemann.pdf (20.02.2014).

Tödtling, F.; Lehner, P.; Trippl, M. (2006): Innovation in knowledge intensive industries: The Nature and Geography of knowledge links. In: European Planning Studies 14, 8, 1035-1058.

Torre, A. (2008): On the role played by temporary geographical proximity in knowledge transmission. In: Regional Studies 42, 6, 869-889.

Trippl, M. (2013): Islands of Innovation as Magnetic Centres of Star Scientists? Empirical Evidence on Spatial Concentration and Mobility Patterns. In: Regional Studies 47, 2, 229-244.

UNESCO - United Nations Educational, Scientific and Cultural Organization (2005): Towards knowledge societies. Paris.

Urry, J. (2002): Mobility and Proximity. In: Sociology 36, 2, 255-274.

Voß, G. G. (2010): Subjektivierung und Mobilisierung. Und: Was könnte Odysseus zum Thema „Mobilität“ beitragen? In: Götz, I.; Lemberger, B.; Lehnert, K.; Schondelmayer, S. (Hrsg.): Mobilität und Mobilisierung. Arbeit im sozioökonomischen, politischen und kulturellen Wandel. Frankfurt a. M., 95-136. = Schriftenreihe Arbeit und Alltag, Band 1.

Weichhart, P. (2009): Multilokalität - Konzepte, Theoriebezüge und Forschungsfragen. In: Informationen zur Raumentwicklung $1 / 2$ (2009), 1-14.

Wiberg, M. (2005): „Anytime, Anywhere“ in the Context of Mobile Work. In: Khosrow-Pour, M. (Hrsg.): Encyclopaedia of Information Science and Technology. Medford, 131-134. 